\title{
A low-cost acoustic permeameter
}

\author{
Stephen A. Drake ${ }^{1}$, John S. Selker ${ }^{2}$, and Chad W. Higgins ${ }^{2}$ \\ ${ }^{1}$ CEOAS, Oregon State University, Corvallis, OR 97333, USA \\ ${ }^{2}$ BEE, Oregon State University, Corvallis, OR 97333, USA
}

Correspondence to: Stephen A. Drake (sdrake@ ceoas.oregonstate.edu)

Received: 19 April 2016 - Discussion started: 19 July 2016

Revised: 4 March 2017 - Accepted: 18 March 2017 - Published: 19 April 2017

\begin{abstract}
Intrinsic permeability is an important parameter that regulates air exchange through porous media such as snow. Standard methods of measuring snow permeability are inconvenient to perform outdoors, are fraught with sampling errors, and require specialized equipment, while bringing intact samples back to the laboratory is also challenging. To address these issues, we designed, built, and tested a low-cost acoustic permeameter that allows computation of volumeaveraged intrinsic permeability for a homogenous medium. In this paper, we validate acoustically derived permeability of homogenous, reticulated foam samples by comparison with results derived using a standard flow-through permeameter. Acoustic permeameter elements were designed for use in snow, but the measurement methods are not snow-specific. The electronic components - consisting of a signal generator, amplifier, speaker, microphone, and oscilloscope - are inexpensive and easily obtainable. The system is suitable for outdoor use when it is not precipitating, but the electrical components require protection from the elements in inclement weather. The permeameter can be operated with a microphone either internally mounted or buried a known depth in the medium. The calibration method depends on choice of microphone positioning. For an externally located microphone, calibration was based on a low-frequency approximation applied at $500 \mathrm{~Hz}$ that provided an estimate of both intrinsic permeability and tortuosity. The low-frequency approximation that we used is valid up to $2 \mathrm{kHz}$, but we chose $500 \mathrm{~Hz}$ because data reproducibility was maximized at this frequency. For an internally mounted microphone, calibration was based on attenuation at $50 \mathrm{~Hz}$ and returned only intrinsic permeability. We found that $50 \mathrm{~Hz}$ corresponded to a wavelength that minimized resonance frequencies in the acoustic tube and was also within the response limitations of the microphone. We used reticulated foam of known per-
\end{abstract}

meability (ranging from $2 \times 10^{-7}$ to $3 \times 10^{-9} \mathrm{~m}^{2}$ ) and estimated tortuosity of 1.05 to validate both methods. For the externally mounted microphone the mean normalized standard deviation was $6 \%$ for permeability and $2 \%$ for tortuosity. The mean relative error from known measurements was $17 \%$ for permeability and $2 \%$ for tortuosity. For the internally mounted microphone the mean normalized standard deviation for permeability was $10 \%$ and the relative error was also $10 \%$. Permeability determination for an externally mounted microphone is less sensitive to environmental noise than is the internally mounted microphone and is therefore the recommended method. The approximation using the internally mounted microphone was developed as an alternative for circumstances in which placing the microphone in the medium was not feasible. Environmental noise degrades precision of both methods and is recognizable as increased scatter for replicate data points.

\section{Introduction}

Intrinsic permeability is the proportionality constant in Darcy's law that describes the interconnectedness of air space in permeable media such as snow. Darcy's law is a constitutive equation that describes fluid flow through a permeable medium and can be written as

$k=\frac{Q L \mu}{\Delta P A}$,

where $k$ is intrinsic permeability $\left(\mathrm{m}^{2}\right), Q$ is volumetric discharge $\left(\mathrm{m}^{3} \mathrm{~s}^{-1}\right), A$ is the sample surface area $\left(\mathrm{m}^{2}\right), L$ is the sample height $(\mathrm{m}), \Delta P$ is the pressure drop $(\mathrm{Pa})$, and $\mu$ is dynamic air viscosity $\left(\mathrm{kg} \mathrm{m}^{-1} \mathrm{~s}^{-1}\right)$. From Eq. (1) it is clear that intrinsic permeability is directly proportional to the flux 
of a fluid through permeable media. Intrinsic permeability has long been recognized as an important parameter that regulates air exchange both within the snow pore space and between the atmosphere and the snowpack (Bader, 1939). Since permeability is difficult to measure directly, early efforts focused on developing empirical relationships that describe permeability as a function of more easily measured parameters such as snow density (Bender, 1957; Shimizu, 1970; Martinelli Jr., 1971). These convenient formulations may be accurate for the specific snow conditions used to establish the empirical formula but often fail for other snow conditions (Domine et al., 2013). In an effort to obtain more accurate field measurements of permeability, several experimental efforts have employed vacuum flow-through devices (Conway and Abrahamson, 1984; Chacho and Johnson, 1987; Hardy and Albert, 1993; Albert et al., 2000; and Courville et al., 2007). However, flow-through measurements are time consuming and fraught with potential sampling errors and alteration of the sample from its native condition (Sommerfeld and Rocchio, 1993). Some sampling issues have been resolved by sample extraction and examination in a lab environment to measure pore space in terms of specific surface area by X-ray tomography and gas adsorption techniques (Kerbrat et al., 2008). But this multi-step process requires specialized equipment and still does not resolve issues of small-scale spatial heterogeneity and sample size representativeness (Albert, 2001).

In this paper, we describe the design, construction, and calibration of an in situ active acoustic device that samples a measurement volume larger than that obtainable with current flow-through devices to acquire a volume-averaged estimate of intrinsic permeability. Acoustically derived permeability measurements are validated with homogenous foam samples of known permeability. Active sampling methods of snow properties have been successfully applied in previous studies. For example, Albert et al. (2007) measured the evolution of an acoustic pulse, generated by firing a pistol blank, and compared the pulse shape with that of a simulated pulse to estimate volume-averaged permeability of an Alaskan snowpack. Kinar and Pomeroy designed (2007) and improved (2015) upon an active acoustic device that infers properties such as snow water equivalent from the differential backscatter at frequencies between $20 \mathrm{~Hz}$ and $10 \mathrm{kHz}$. The two methods that we present are less sophisticated than either the Albert or Kinar methods but have the advantage that no specialized equipment is required. Our design borrows elements from Ishida (1965), Buser (1986), and Moore et al. (1991).

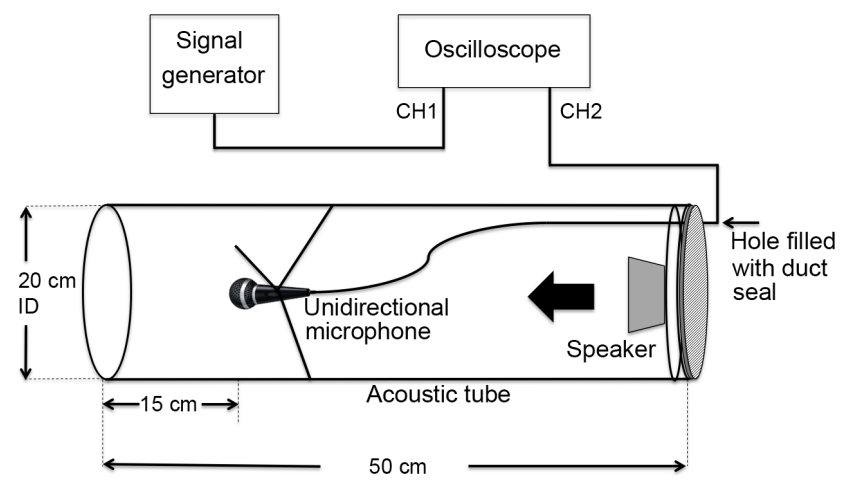

Figure 1. Acoustic permeameter schematic.

\section{Method}

\subsection{Design and assembly of the acoustic permeameter}

We assembled an acoustic permeameter from commonly available parts (Fig. 1). A Heathkit Model IG-1275 signal generator produced a sine wave of a specified frequency and amplitude that was split and directed both to channel 1 of a Tektronix TDS 1001 oscilloscope and to a $4 \Omega$ Altec A4468 speaker. Electrical components were powered from a DC$\mathrm{AC}$ inverter and $12 \mathrm{~V}$ car battery. The speaker was screwmounted onto a ring of 3/4 in. thick medium-grade plywood. An acoustic tube was constructed using $20 \mathrm{~cm}$ (inside) diameter, schedule-40 PVC pipe. The acoustic tube was vertically mounted onto the plywood ring and secured with butterfly clamps. During measurement, the tube was placed on the medium of interest with the speaker facing downward such that sine waves emanating from the speaker would interrogate the medium. The subsequent acoustic response was captured by a Radio Shack ${ }^{\mathrm{TM}}$ unidirectional microphone (model 3303038; frequency range: $50 \mathrm{~Hz}-15 \mathrm{kHz}$ ) and directed to the second input channel on the oscilloscope. Frequency and amplitude of input and output signals were stored on the oscilloscope USB drive. We do not have full knowledge of the characteristics of the microphone transducer. However, the calibration is empirical and will vary with each microphone, so discrepancies between microphones are accommodated by the calibration.

\subsection{Acoustic permeability determination - external microphone method}

The first method used to measure intrinsic permeability involved using an externally placed microphone (EM), where we placed the test media between the sound source and the microphone (Fig. 2). The calibration setup for the EM method had the speaker mounted in an upward orientation and the microphone mounted directly above the centerline of the PVC tube, facing downward. Acoustic intensity at a given frequency was measured with the microphone in open 


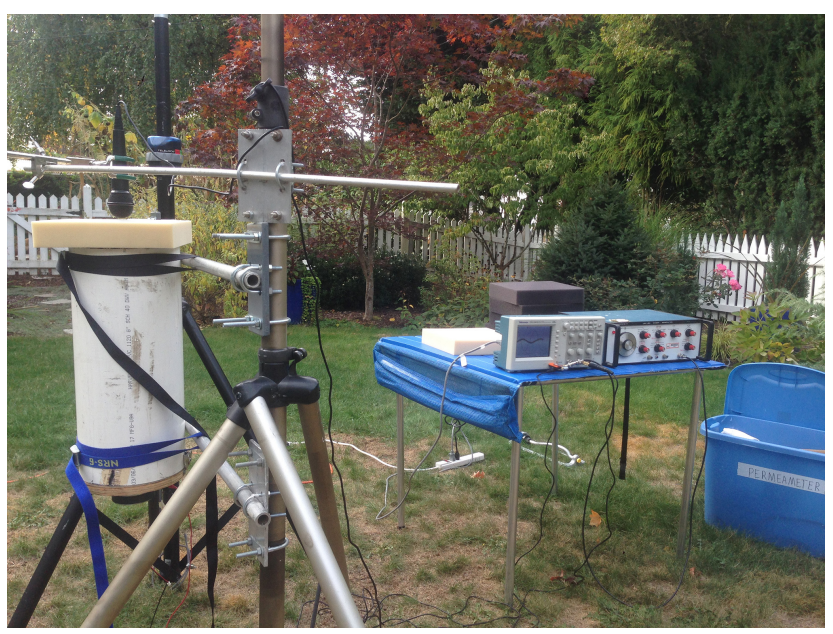

Figure 2. EM calibration setup with the microphone suspended above a foam sample. Foam samples, the oscilloscope, and the frequency generator are located on the foldup table. Not shown are a ring weight that was placed around the perimeter of the foam sample to minimize vibration and foam sheets that were placed beneath the acoustic tube to dampen amplitude of reflected acoustic energy.

air to establish reference amplitude. Then a foam sample was placed over the end of the speaker tube and weighted at the edges to minimize sample vibration. At each applied frequency, attenuation and phase shift were determined by comparison with the reference measurement. We then utilized the low-frequency approximation in Moore et al. (1991, hereafter referred to as M91) to calculate permeability. In M91, estimates of tortuosity $(\tau)$ and effective flow resistivity $\left(\sigma_{\mathrm{pe}}\right)$ were iteratively adjusted until optimal agreement between the measured and modeled value for the propagation constant $\left(k_{b}\right)$ at a given frequency $(f)$ was obtained. Snow permeability $(k)$ can then be computed from flow resistivity given knowledge or assumptions of the snow grain shape. The theoretical basis for the M91 low-frequency approximation is described in Sect. 3.1 of this paper. An added benefit of this method relative to a flow-through permeameter is that it produces a measure of tortuosity as well as permeability.

\subsection{Acoustic permeability determination - internal microphone method}

A second permeability measurement method employed the microphone mounted inside the acoustic tube, facing outward, at a fixed position $(15 \mathrm{~cm})$ from the open end. We refer to this as the IM method. We measured attenuation at $50 \mathrm{~Hz}$ relative to an open-air value with the open end of the acoustic tube snug against the test media. At this frequency, the wavelength of the emitted frequency is much greater than the length of the acoustic tube. Since the medium represents an acoustic barrier to the emitted waveform, the maximum amplitude of the transmitted waveform was progressively retarded as permeability increased. Simultaneously, the ampli-

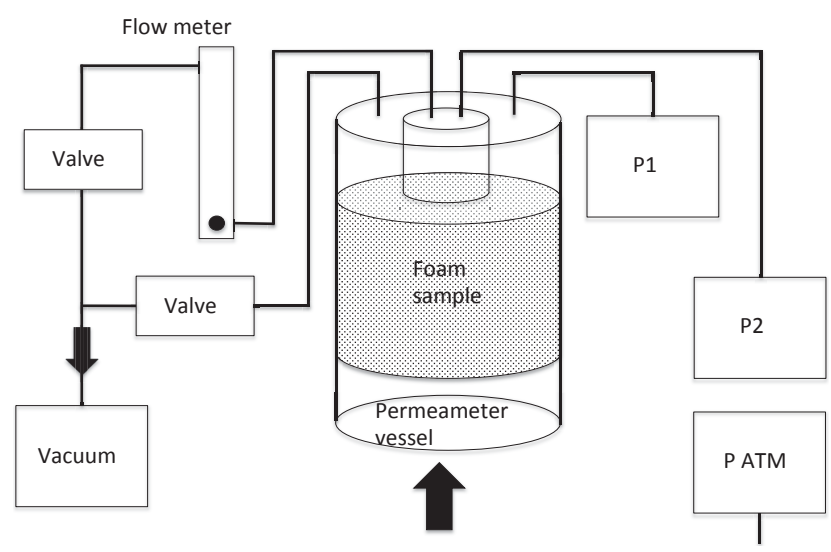

Figure 3. Flow-through permeameter with three absolute pressure sensors labeled P1, P2, and P ATM. P ATM measures atmospheric pressure. This permeameter design is a modified version of the flowthrough permeameter of Hardy and Albert (1993).

tude of the reflected waveform, measured by the internally mounted microphone, increased. From Morse (1952, hereafter referred to as M52), attenuation at a given frequency is a function of flow resistivity. Amplitude of the reflected waveform is inversely proportional to the amplitude of the transmitted waveform, so permeability was computed as a function of the ratio between the amplitude of the imposed waveform and the reflected amplitude.

\subsection{Flow-through permeability determination}

We verified acoustic permeameter measurements with $5 \mathrm{~cm}$ thick reticulated foam samples. The permeability of these foam samples was established with a flow-through permeameter similar to Albert et al. (2000) with the exception that we utilized three high-precision (Paroscientific 216B) absolute pressure sensors rather than two relative pressure sensors (Fig. 3). Using absolute pressure sensors rather than relative pressure sensors required a modification of Albert's method, so we briefly describe our alternate method. For each foam type we cut a cylindrical section and spread a layer of petroleum gel around the cut edge. We then slid the cut foam into the double-walled permeameter until the inner face of the foam was flush against the inner wall of the permeameter as shown in Fig. 3. The gel sealed gaps between the foam and vessel wall. We cross-calibrated the three pressure sensors before initiating airflow through the permeameter. The three pressure sensors monitored atmospheric, inner-cylinder, and outer-cylinder pressures. Following Albert et al. (2000), we acquired the pressure drop across each foam sample at 8-12 flow rates while adjusting a valve to regulate the pressure between the inner and outer vessel to eliminate radial flow. We used the slope of the subsequent flow rate vs. pressure drop curve to derive permeability from Darcy's law valid for Reynolds numbers less than 1. Our samples were distinct in properties from those published by 
Table 1. Regicell foam permeability from Clifton et al. (2008) and FXI foam permeability measured with a flow-through permeameter described in this paper.

\begin{tabular}{ll}
\hline Foam type & Flow-through permeability $\left(\mathrm{m}^{2}\right)$ \\
\hline Regicell 10 & $160 \times 10^{-9}$ \\
Regicell 30 & $39 \times 10^{-9}$ \\
Regicell 60 & $6 \times 10^{-9}$ \\
FXI Z10FRB & $206 \times 10^{-9}$ \\
FXI Z20FRB & $137 \times 10^{-9}$ \\
FXI Z30FRB & $47 \times 10^{-9}$ \\
FXI Z50M & $15 \times 10^{-9}$ \\
FXI Z80CLE & $3 \times 10^{-9}$ \\
\hline
\end{tabular}

Clifton et al. (2008) (Table 1), suggesting that intrinsic permeability of reticulated foam with the same specified pores per linear inch (PPI) but different manufacturers (here Regicell and FXI Corporation) must be determined experimentally. Permeability measured for the FXI samples in Table 1 span expected snow permeability values ranging from lightly compacted snow (low permeability) to depth hoar (high permeability) (Arakawa et al., 2009).

\section{Theory}

In this section, we describe the theoretical basis for the two methods used to acoustically measure snow permeability.

\subsection{Theory for the EM method}

The complex propagation constant for sound through permeable media can be written in general terms as

$k_{b}=a+i b$.

Coefficients of the propagation constant define the phase and magnitude of attenuation, respectively, and are defined in M91 as

$a=\frac{\pi \Delta \phi}{180 d}$,

$b=\frac{\text { Attenuation }(d B) \ln (10)}{20 d}$,

where $\Delta \phi$ is the phase shift between a free-air measurement and the in-snow measurement, and $d$ is media thickness. We measure attenuation in terms of a voltage difference between the input $\left(V_{\mathrm{IN}}\right)$ and output signal $\left(V_{\mathrm{OUT}}\right)$ as

Attenuation $(d B)=20 \log _{10}\left(\frac{V_{\mathrm{OUT}}}{V_{\mathrm{IN}}}\right)$.

Attenborough (1983) developed a four-parameter acoustic model that describes acoustic attenuation through permeable media. These four parameters - porosity, effective flow resistivity, pore shape factor, and grain shape factor - collapse to three parameters in the low-frequency approximation given in M91:

$k_{b}=0.0079 \sqrt{f}\left[9.10 \tau f+i 4 \sigma_{\mathrm{pe}}\right]^{0.5}$,

where, as in M91, the constant 9.10 is derived by assuming that the speed of sound is air is $328 \mathrm{~m} \mathrm{~s}^{-1}$ and air density is $1.317 \mathrm{~kg} \mathrm{~m}^{3}$ at $1013 \mathrm{kPa}$ and $-5^{\circ} \mathrm{C}$ air temperature. For snow, this low-frequency approximation (Eq. 6) is valid at frequencies below $2 \mathrm{kHz}$. The M91 authors used Eq. (6) to calculate the propagation constant for a given tortuosity and flow resistivity and Eqs. (2)-(5) to calculate the propagation constant from phase shift and attenuation. Values for flow resistivity and tortuosity in Eq. (6) were iteratively adjusted until the difference between the propagation constant determined by these two methods was minimized. Due to environment-dependent nonlinear responses at both low and high frequencies, the M91 authors obtained the best fit when they used well-chosen point measurements rather than measurements over a range of frequencies. Similarly, our results are based on well-chosen point measurements. But instead of iteratively solving Eq. (6), we separate the real and imaginary parts to independently solve for tortuosity and effective flow resistivity:

$\tau=\frac{\left[a^{2}-b^{2}\right]}{[9.10][0.0079]^{2} f^{2}}$,

$\sigma_{\mathrm{pe}}=\frac{a b}{2[0.0079]^{2} f}$.

We then apply empirical calibrations to match derived tortuosity and effective resistivity with known values. Empirical calibrations are needed because the reference amplitude is not measured by a reference microphone but is rather the amplitude applied by the frequency generator.

\subsection{Theory for the IM method}

With the alternate configuration of the microphone placed inside the acoustic tube, Eqs. (2)-(8) are no longer applicable. Instead, we use an alternate theoretical basis to determine intrinsic permeability based on results described in M52. M52 found and Ishida (1965) verified a simple relationship between flow resistivity and signal attenuation:

$\alpha=\beta \sqrt{\omega \sigma} \rightarrow \sigma=\frac{\alpha^{2}}{2 \pi f} \times 10^{n}$,

where $\alpha$ is an attenuation constant, $\beta$ is an empirical constant, $\omega$ is the imposed angular frequency, and $\sigma$ is flow resistivity. As with the EM method, we fit a sine wave to the input and output signals to eliminate environmental and 
microphone-derived noise and then determined the phase offset and attenuation between the input and output signals as a function of the input frequency. Instead of measuring the attenuation through the media, we measure the amplitude of the reflected waveform at $50 \mathrm{~Hz}$, a midpoint value for the low-frequency approximation. We take the amplitude of the reflected acoustic energy as an empirically derived function of transmitted acoustic energy. Using reticulated foam samples of known permeability, we relate the ratio of the reflected signal amplitude to initial signal amplitude with flowthrough permeability measurements.

\section{Results}

\subsection{Results for the EM method}

Having calibrated permeability of the reticulated foam samples with the flow-through permeameter (in Fig. 3), we subsequently obtained acoustic measurements by the EM method. Three replicates were acquired for each foam sample at each frequency. After some experimentation, we opted to utilize data acquired at $500 \mathrm{~Hz}$ because this frequency lies within the valid range of frequencies (up to $2 \mathrm{kHz}$ ) for the low-frequency approximation and because the $500 \mathrm{~Hz}$ results were more replicable than those acquired at higher frequencies. The setup for this phase of the experiment is shown in Fig. 2.

In M91 flow resistivity and tortuosity were determined by iteratively finding the best fit between measurements given in Eqs. (2)-(5) and the theoretical prediction given in Eq. (6). Since we know a priori the foam tortuosity and permeability, we compared the known effective flow resistivity with the effective flow resistivity computed from Eqs. (2)-(8). As in Albert et al. (2007) and using a pore shape factor ratio $\left(s_{\mathrm{f}}\right)$ of 1 , the effective flow resistivity as derived from permeability is

$\sigma_{\mathrm{pe}} s_{\mathrm{f}}^{2}=\frac{\mu}{k}$.

For the foam samples, we found that a linear fit resolved the difference between computed and known flow resistivity for a given permeability. In short, acoustically derived effective flow resistivity did not match the actual effective flow resistivity but deviated by a linear function from it. Therefore, from Eq. (10), we computed flow resistivity as a linear function of permeability.

In Fig. 4 we plotted the results of three independent data sets, with each data set corresponding to a different volume setting on the signal generator and rendered in a different color. For a given data set, the signal generator volume was established for the first data point and not changed for the remaining data points. Data corresponding to the blue, black, and red data sets had initial imposed volumes that were associated with root mean square (rms) signal input voltages of 1.3, 2.3, and 5.6 V, respectively. Plotted in $\log -\log$ format,

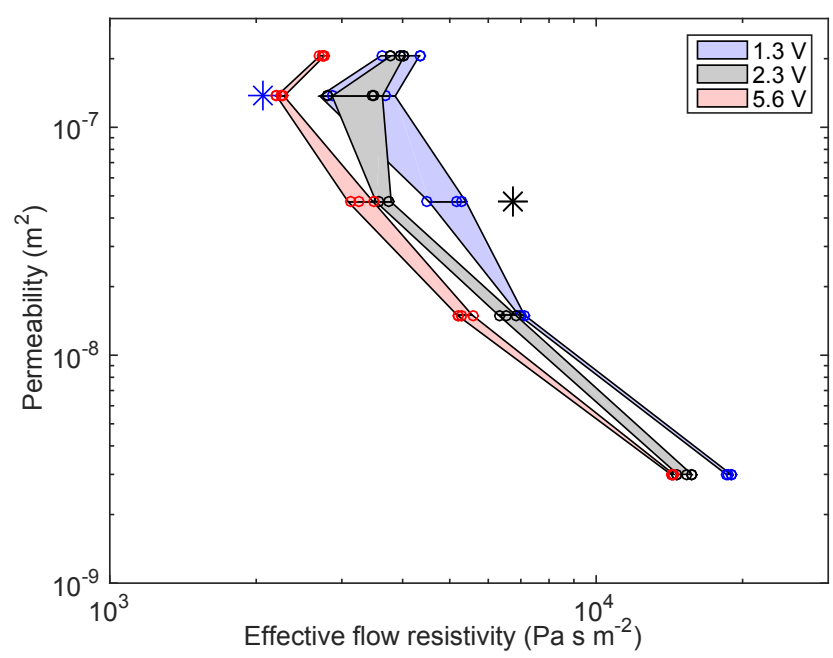

Figure 4. Comparison of acoustically derived permeability using the M91 method at $500 \mathrm{~Hz}$ and three different initial conditions. The three initial conditions correspond to input rms signal voltages of 1.3, 2.3, and 5.6 volts highlighted in blue, black, and red, respectively. Data were acquired outdoors on clear days during periods of light winds. Color-filled regions indicate data within 1 standard deviation of the mean. The asterisks indicate data points that exceeded 1 standard deviation from the mean (before they were excluded) and were thus excluded from subsequent analysis.

data points for each data set in Fig. 4 are roughly collinear, and the slope of the best-fit line for a given data set is nearly parallel to the other two data sets, but the data sets are not coincident (Fig. 5). This result indicates that, as the amplitude of the imposed sine wave was increased, absolute attenuation of the signal also increased. Since the acoustically derived permeability is sensitive to the amplitude of the imposed waveform, it is therefore important to use the same amplitude waveform in the calibration as in subsequent permeability measurements. The relationship between $\sigma_{\text {pe }}$ and $k$ reversed at the highest permeability in Fig. 4. The reason for this reversal is possibly attributable to nonlinearity in microphone response and represents an upper limit to the permeability that can be measured by this technique within the constraints of our experimental design. Triplicate data points were nearly coincident in most cases; however, the blue and black asterisks in Fig. 4 correspond to points that were more than a standard deviation removed from the other two points in the given triplicate measurement. Differences in the imposed amplitude for these two data points do not account for their deviation from the mean, suggesting the source of error was environmental. These data were acquired outdoors in a relatively quiet but acoustically uncontrolled location, so we attribute this error to intermittent external noise. It is therefore also important to acquire multiple data points for each measurement so that one can distinguish the impact of incidental external noise. 


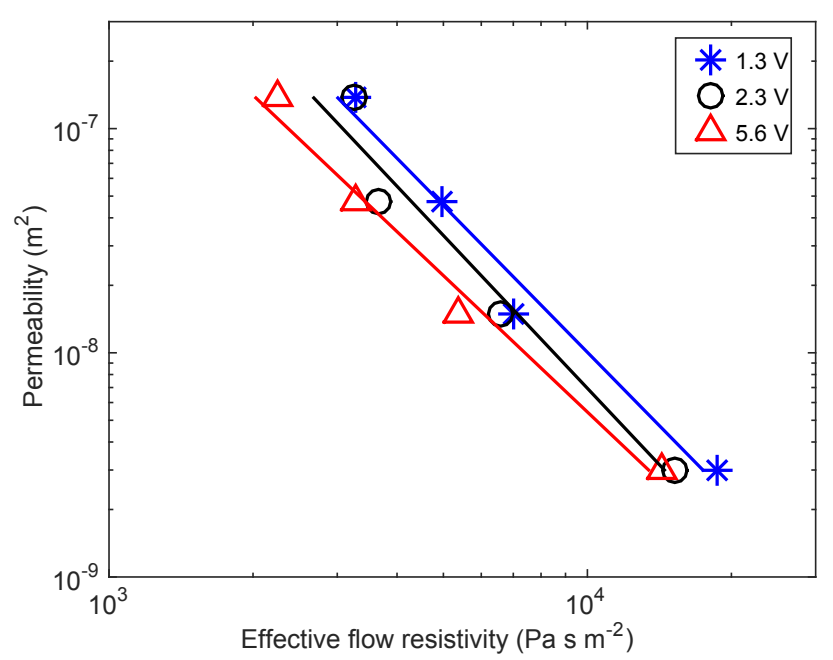

Figure 5. Linear regressions for data in Fig. 4 with the same color for each data set. $R^{2}$ values were 0.98 (blue), 0.98 (black), and 0.99 (red).

Uncalibrated tortuosity calculated for different foam samples was nearly very similar but unrealistically high with an average of 17.7 and standard deviation of 1.54 between all of the samples. A slight but monotonic decrease in measured tortuosity with decreasing permeability accounted for most of the standard deviation as opposed to random scatter. For comparison, analogous data from Álvarez-Arenas et al. (2006), Kino et al. (2012), and references in Melon and Castagnede (1995) and Doutres and Atalla (2012) note that reticulated foam commonly has a narrow range of tortuosity from 1.03 to 1.06 . Tortuosity of the FXI-reticulated foam is not independently measured but shares other specifications with these other sources and likely has tortuosity in the same range.

We empirically compensate for the differences between the theoretical and experimentally derived values for tortuosity and effective flow resistivity. Our rationale is that the functional relationships between the theoretically and derived values for tortuosity and effective flow resistivity are the same, so the differences in values can be resolved by a first-order calibration. Calculated tortuosity depended very weakly on media permeability, so multiplying the calculated tortuosity by an empirically derived constant renders the theoretical value. On the other hand, calculated permeability varies linearly (on a log scale) with effective flow resistivity (as shown in Fig. 5), so a linear calibration is required to calculate permeability from measured effective flow resistivity. The multiplicative constant for tortuosity is found by dividing the mean known tortuosity by the mean derived tortuosity. The linear calibration relating effective flow resistivity to permeability is taken from the slope of the line in Fig. 5. In practice, one must calibrate the acoustic permeameter with media of known permeability and tortuosity to derive these multiplicative constants. Once these multiplica-

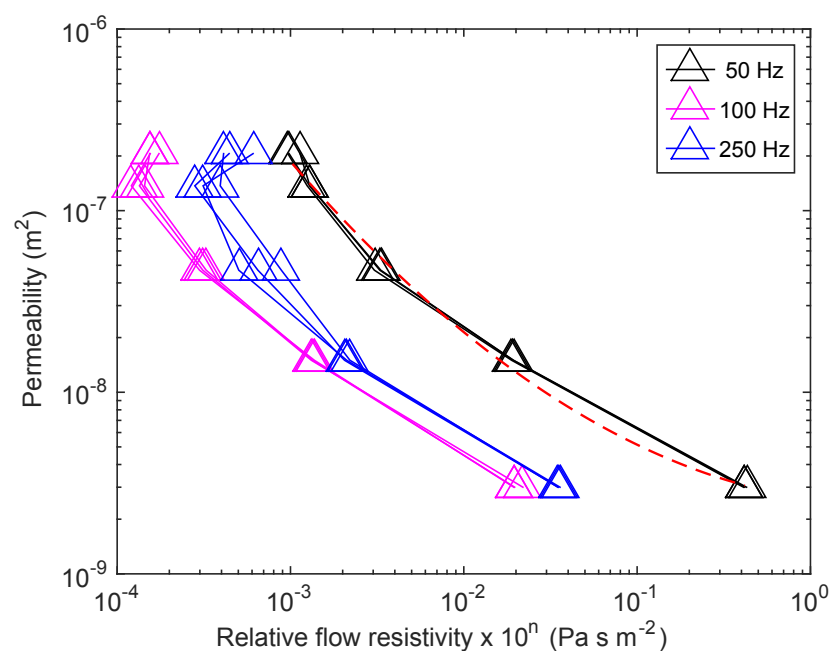

Figure 6. Flow-through permeability as a function of relative flow resistivity using the M52 method and Eq. (9) at 50, 100, and $250 \mathrm{~Hz}$. Three data points were acquired at each frequency and each foam type. The dashed red line defines a second-order polynomial curve fit for the measurements with $k=10^{\left[0.16(\log \sigma)^{2}-0.14(\log \sigma)-8.59\right]}$.

tive constants have been determined for the acoustic permeameter, the tortuosity and permeability of other media having similar impedance can be derived without a priori knowledge of media tortuosity or permeability. For the $5.6 \mathrm{~V}$ data set we find the multiplicative constant as $\alpha=0.057$. For the same data set a linear fit between measured flow resistivity and known permeability is given by

$\log _{10}(k)=-2.0219 \times \log _{10}\left(\sigma_{\mathrm{pe}}\right)-0.1764$,

With these modifications, the M91 method obtains derived tortuosity ranging from 1.02 to 1.07 for the foam samples with a standard deviation of \pm 0.03 . The normalized standard deviation percentage for permeability measurements ranged from $2.1 \%$ for the Z80 foam to $11.6 \%$ for the Z30 foam with a mean of $6.2 \%$ across all foam samples. We normalize the standard deviation because this error measure does not change when effective flow resistivity is calibrated with a multiplicative constant. The average percentage error between known and derived permeability was $17 \%$ by the EM method.

\subsection{Results for the IM method}

Applying Eq. (9) to results obtained with the microphone placed inside the acoustic tube at 50,100, and $250 \mathrm{~Hz}$ yielded results shown in Fig. 6. Since we are measuring reflected acoustic energy rather than transmitted energy, the result shown in Fig. 6 is an indirect (relative) measure of flow resistivity. Triplicates were acquired for each foam sample, and these data were highly reproducible as shown by the close grouping of each triplicate. Data at these three frequencies were acquired on different days, each with a slightly different 
amplitude baseline (free-air) setting, accounting for the horizontal displacement between curve fits. The normalized standard deviation percentage for $50 \mathrm{~Hz}$ measurements ranged from $0.45 \%$ for the Z50 sample to $19 \%$ for the highestpermeability (Z10) sample, with a mean of $10 \%$ across all foam samples. The average percentage error between known and derived permeability was $10 \%$ by the IM method. Since data at $50 \mathrm{~Hz}$ show improved precision relative to 100 and $250 \mathrm{~Hz}$ data, as given by the wider domain of reflected signal amplitude, we use data at $50 \mathrm{~Hz}$ to compute permeability. The piecewise spline fit (black, in Fig. 6) describes the straight-line fit between acoustically derived resistivity, and the red dashed line delineates a smooth curve used to calibrate measurements to flow-through permeability. There is a measurable phase shift with decreasing permeability of the test media; however, M52 does not offer a relationship for tortuosity, so none was attempted with this method. Microphone response limitations precluded measurements below $50 \mathrm{~Hz}$.

\section{Discussion}

Derived permeability using the EM and IM methods is compared with the flow-through (known) permeability in Fig. 7. Intrinsic permeability for each foam sample is given in Table 1. Ideally, all measurements would fall on the diagonal line in Fig. 7, meaning that acoustically measured permeability equals known permeability for each foam sample. We find satisfactory agreement with known permeability for both acoustic methods. The average percentage error for the EM method was 17 and $10 \%$ for the IM method. However, the curve fit for the IM method is nonlinear, so a small error in effective flow resistivity for a high-permeability medium has a greater impact than the same error for a low-permeability medium.

The EM and IM methods for determining permeability each have advantages and disadvantages. As mentioned, one advantage of the EM method is that the function relating effective flow resistivity to permeability is linear, unlike the more complicated relationship for the IM method. An advantage of a linear function is that a small error in measuring the independent variable yields an error that is invariant with relative magnitude when determining the dependent variable, regardless of the magnitude of the measurement. This behavior is not true for nonlinear functions such as the more complicated function employed in the IM method. A disadvantage of the EM method is that it is not valid for highpermeability media over the range of parameters that we tested (e.g., $5 \mathrm{~cm}$ media thickness). The IM method suffers decreasing precision at high permeability because the slope of the curve is increasing, but, unlike the EM method, the curve fit remains valid. Data acquired using the IM method were highly reproducible for all of our samples, which to some extent compensates for the slope-induced decrease in

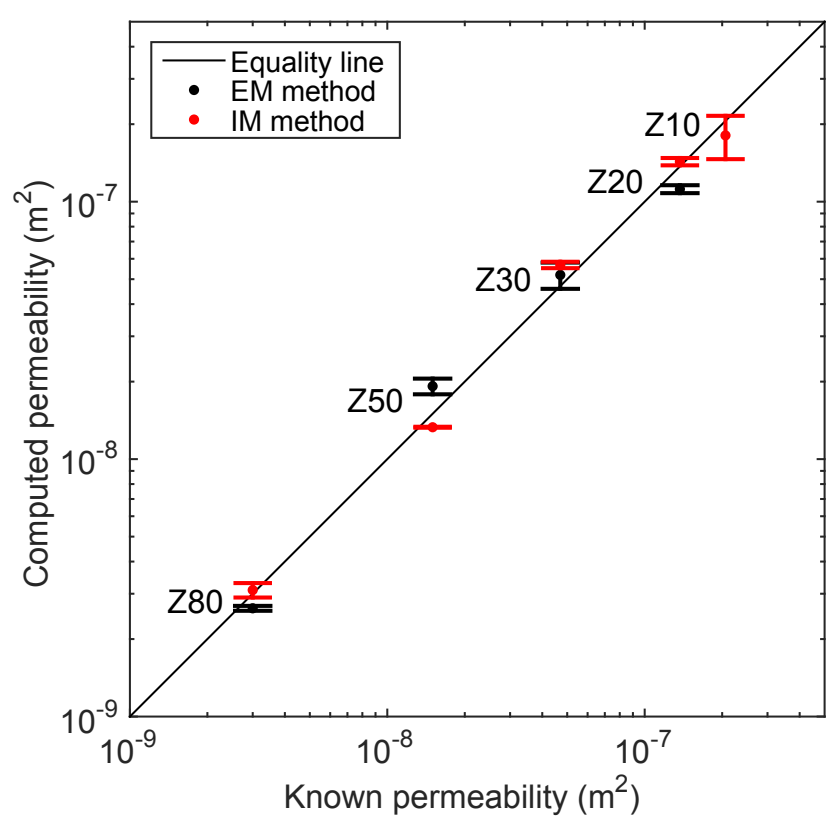

Figure 7. Comparing flow-through (known) permeability with the EM method at $500 \mathrm{~Hz}$ and the IM method at $50 \mathrm{~Hz}$ for foam samples given in Table 1 . Z10 permeability was not computed by the EM method because $k / \sigma_{\text {pe }}$ is nonlinear at high permeability, as shown in Fig. 4. For both methods, the value for each permeability measurement is given from the curve fit for that method, and the standard deviation is given from the original measurements.

precision at high permeability. Another potential disadvantage of the EM method is that the microphone must be placed in the media to be measured. Depending on the circumstance, emplacement in the media may be difficult or impractical. Finally, a disadvantage of the IM method is that it is more empirical than the EM method, which may limit its applicability to different media types. Due to the linear functional form of the EM method, we suggest that it is the preferred method as long as the media to be measured have permeability that is within the valid range of measurements given by the calibration.

The results presented in this paper apply directly to reticulated foam, which has similar microphysical characteristics to snow (Schneebeli and Sokratov, 2004; Clifton et al., 2008). Nevertheless, foam is not snow, and we have yet to establish how differences in characteristic impedance between reticulated foam and snow will affect signal attenuation measurements. The next step is to validate acoustically derived measurements in snow by comparison with other methods. Given that characteristic impedance of snow varies linearly with snow density (Marco et al., 1998), we anticipate that the calibration curves for both the EM and IM methods will have similar shape to the curves in Figs. 5 and 6 but with a modified slope for snow. 


\section{Conclusions}

We conclude that it is feasible to derive intrinsic permeability with a low-cost acoustic permeameter by either the EM method or the IM method. This system returns an unambiguous, volume-averaged permeability consistent with snow densities ranging from lightly compacted snow to depth hoar. The EM method returned intrinsic permeability within $17 \%$ of flow-through measurements and within $2 \%$ of comparable tortuosity measurements. The IM method returned intrinsic permeability within $10 \%$ of flow-through measurements. Measurement reproducibility by the IM method is very high, such that the standard deviation between measurements is dwarfed by environmental factors such as background noise or inhomogeneities in the media of interest. These methods are relevant in studies for which an expedient volume average trumps a more detailed but time-consuming vertical profile that requires measuring multiple samples using a flow-through permeameter. One significant advantage of using these methods is that a particular frequency is measured rather than a broad spectrum. This feature obviates the need for specialized equipment used by other methods, such as a random noise generator and a spectral recorder/analyzer. Disadvantages of this system are that measurements are affected by environmental conditions such as background noise and wind. A future task is to validate this system by comparison with other measures of intrinsic permeability over a range of snow habits and densities.

Data availability. Data and data file descriptions are available online through the Scholars Archive hosted by Oregon State University with reference doi:10.7267/N95H7D65 (Drake et al., 2017).

Competing interests. The authors declare that they have no conflict of interest.

Acknowledgements. We thank FXI Corporation for providing the reticulated foam samples that we used to perform the permeability calibrations. Thanks also to Ziru Liu and Rebecca Hochreutener for their assistance in acquiring data.

Edited by: C. Waldmann

Reviewed by: N. J. Kinar and one anonymous referee

\section{References}

Albert, D. G.: Acoustic waveform inversion with application to seasonal snow covers, J. Acoust. Soc. Am., 109, 91-101, doi:10.1121/1.1328793, 2001.

Albert, D. G., Decato, S. N., and Carbee, D. L.: Snow cover effects on acoustic sensors, Cold Reg. Sci. Technol., 52, 132-145, doi:10.1016/j.coldregions.2007.05.009, 2007.
Albert, M. R., Shultz, E. F., and Perron Jr., F. E.: Snow and firn permeability at Siple Dome, Antarctica, Ann. Glaciol., 31, 353356, doi:10.3189/172756400781820273, 2000.

Álvarez-Arenas, T. E. G., de la Fuente, S., and Gómez, I. G.: Simultaneous determination of apparent tortuosity and microstructure length scale and shape: Application to rigid open cell foams, Appl. Phys. Lett., 88, 221910, doi:10.1063/1.2208921, 2006.

Arakawa, H., Izumi, K., Kawashima, K., and Kawamura, T.: Study on quantitative classification of seasonal snow using specific surface area and intrinsic permeability, Cold Reg. Sci. Technol., 59, 163-168, doi:10.1016/j.coldregions.2009.07.004, 2009.

Attenborough, K.: Acoustical characteristics of rigid fibrous absorbents and granular materials, J. Acoust. Soc. Am., 73, 785799, doi:10.1121/1.389045, 1983.

Bader, H.: Der Schnee und Seine Metamorphose, Beitrag zur Geologie der Schweiz, Geotechnishe Serie, Hydrologie, Lieferung, 3, Bern, 1939.

Bender, J. A.: Air Permeability of Snow, Research report (U.S. Army Snow, Ice, and Permafrost Research Establishment), 37, 1957.

Buser, O.: A rigid-frame model of porous media for the acoustic impedance of snow, J. Sound Vib., 111, 71-92, doi:10.1016/S0022-460X(86)81424-9, 1986.

Chacho, E. F. and Johnson, J. B.: Air permeability of snow, EOS (Transactions of the AGU), 68, 1271, 1987.

Clifton, A., Manes, C., Ruedi, J. D., Guala, M., and Lehning, M.: On shear driven ventilation of snow, Bound. Lay. Meteorol., 126, 249-261, doi:10.1007/s10546-007-9235-0, 2008.

Conway, H. and Abrahamson, J.: Air permeability as a textural indicator of snow, J. Glaciol., 30, 328-333, doi:10.3198/1984JoG30106-328-333, 1984.

Courville, Z. R., Albert, M. R., Fahnestock, M. A., Cathles, L. M., and Shuman, C. A.: Impacts of an accumulation hiatus on the physical properties of firn at a low-accumulation polar site, J Geophys. Res., 112, F02030, doi:10.1029/2005JF000429, 2007.

Domine, F., Morin, S., Brun, E., Lafaysse, M., and Carmagnola, C. M.: Seasonal evolution of snow permeability under equi-temperature and temperature-gradient conditions, The Cryosphere, 7, 1915-1929, doi:10.5194/tc-7-1915-2013, 2013.

Doutres, O. and Atalla, N.: A semi-empirical model to predict the acoustic behaviour of fully and partially reticulated polyurethane foams based on microstructure properties, in: Proceedings of the Acoustics 2012 Nantes Conference, Nantes, France, 23-27 April 2012, 1956-1960, 2012.

Drake, S. A., Selker, J. S., and Higgins, C. W.: A low-cost acoustic permeameter, OSU Libraries, Dataset, doi:10.7267/N95H7D65, 2017.

Hardy, J. and Albert D.: The permeability of temperate snow: Preliminary links to microstructure, Proc. Eastern Snow Conf., 50, 149-156, 1993.

Ishida, T.: Acoustic properties of snow, Low Temperature Science, Series A: Physical Sciences 20, 23-63, 1965.

Kerbrat, M., Pinzer, B., Huthwelker, T., Gäggeler, H. W., Ammann, M., and Schneebeli, M.: Measuring the specific surface area of snow with X-ray tomography and gas adsorption: comparison and implications for surface smoothness, Atmos. Chem. Phys., 8, 1261-1275, doi:10.5194/acp-8-1261-2008, 2008. 
Kinar, N. J. and Pomeroy, J. W.: Determining snow water equivalent by acoustic sounding, Hydrol. Process., 21, 2623-2640, doi:10.1002/hyp.6793, 2007.

Kinar, N. J. and Pomeroy, J. W.: SAS2: The system for acoustic sensing of snow, Hydrol. Process., 29, 4032-4050, doi:10.1002/hyp.10535, 2015.

Kino, N., Nakano, G., and Suzuki, Y.: Non-acoustical and acoustical properties of reticulated and partially reticulated polyurethane foams, Appl. Acoust., 73.2, 95-108, doi:10.1016/j.apacoust.2011.06.009, 2012.

Marco, O., Buser, O., Villemain, P., Touvier, F., and Revol, P.: Acoustic impedance measurement of snow density, Ann. Glaciol., 26, 92-96, doi:10.3198/1998AoG26-1-92-96, 1998.

Martinelli Jr., M.: Physical properties of alpine snow as related to weather and avalanche conditions, U.S. Dept. Of Agriculture, Forest Service Research Paper RM-64, 1971.

Melon, M. and Castagnede, B.: Correlation between tortuosity and transmission coefficient of porous media at high frequency, J. Acoust. Soc. Am., 98, 1228, doi:10.1121/1.413623, 1995.
Moore, H. M., Attenborough, K., Rogers, J. and Lee, S.: In-situ acoustical investigations of deep snow, Appl. Acoust., 33, 281301, doi:10.1016/0003-682X(91)90018-A, 1991.

Morse, R. W.: Acoustic propagation in granular media, J. Acoust. Soc. Am., 24, 696-700, doi:10.1121/1.1906958, 1952.

Schneebeli, M. and Sokratov, S.: Tomography of temperature gradient metamorphism of snow and associated changes in heat conductivity, Hydrol. Process., 18, 3655-3665, doi:10.1002/hyp.5800, 2004.

Shimizu, H.: Air permeability of deposited snow, Contrib. Inst. Low Temp. Sci. Ser. A, 22, 1-32, 1970.

Sommerfeld, R. A. and Rocchio, J. E.: Permeability measurements on new and equitemperature snow, Water Resour. Res., 29, 2485 2490, doi:10.1029/93WR01071, 1993. 